\title{
CUÁL ES SU DIAGNÓSTICO?
}

\section{Antonio Guglielmetti $\mathbf{V}^{1}$, Manuel Villanueva $\mathrm{F}^{2}$, Ignacio Soto $\mathbf{R}^{2}$}

'Dermatólogo, Departamento de Dermatología, Universidad de Valparaíso, Valparaíso, Chile; ${ }^{2}$ Internos de Medicina, Universidad de Valparaíso, Valparaíso, Chile.

\section{Caso clínico}

Paciente de sexo femenino, con antecedente de lesión dermatológica desde el los 10 años (2003), cuando realiza primera consulta por lesiones papulares eritematosas, ubicadas en región dorsal derecha con patrón zosteriforme, asintomáticas, de aparición progresiva y que en ese momento llevaba 2 años de evolución. Mediante estudio histológico se realizó el diagnóstico de dermatitis nodular profunda, compatible con reacción tardía a picadura de insecto.

Acude a segunda consulta con 21 años de edad (2015) por persistencia y aumento del número de pápulas que confluyen formando una placa eritemato-violácea con patrón linear, siguiendo las líneas de Blaschko en el dorso (Figura 1). La lesión mantiene su carácter asintomático.

Se realiza biopsia que muestra piel con ortoqueratosis y epidermis normal, dermis con infiltración linfoide densa, nodular, superficial, constituida por linfocitos, algunos de núcleo irregular, plasmocitos e histiocitos. No se reconocen granulomas ni eosinófilos. Los anexos se encuentran conservados y la tinción de PAS es negativa para hongos (Figura 2). Se solicita estudio con Inmunohistoquímica que informa reacción positiva para BCL-2 en $80 \%$ de los linfocitos, para BCL-6 en $20 \%$, para cadenas livianas kappa en $50 \%$ de plasmocitos y para cadenas livianas lambda en $50 \%$ de plasmocitos.

Se aplicaron corticoides intralesionales a la paciente (betametasona $1 \mathrm{mg} / \mathrm{ml}$ : 0,2 $\mathrm{ml}$ ), con regresión rápida y significativa de las lesiones en el control 2 semanas después de la consulta.

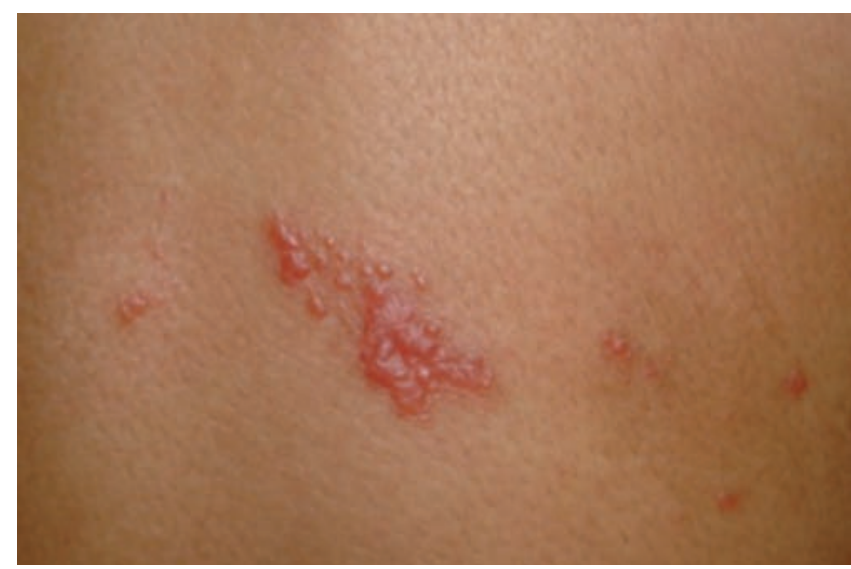

Figura 1: Compromiso dorsal al momento de la segunda consulta. Múltiples pápulas eritemato-violáceas coalescentes en placa.

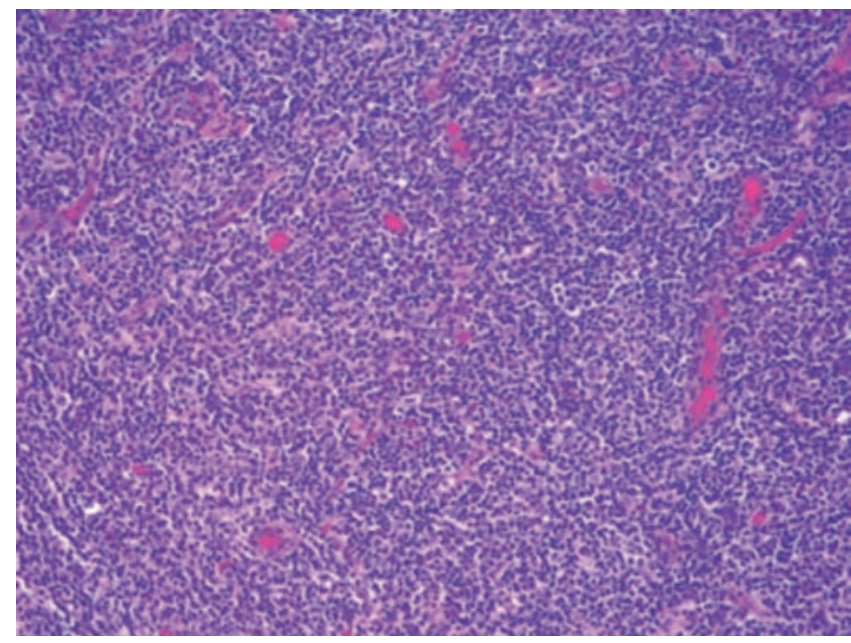

Figura 2: Biopsia de lesión. Infiltración linfoide dérmica densa, nodular, superficial, constituida por linfocitos, algunos de núcleo irregular, plasmocitos e histiocitos. No se reconocen granulomas ni eosinófilos. 


\section{Diagnóstico}

\section{Hiperplasia Linfoide Cutánea}

La Hiperplasia linfoide cutánea (HLC) es un proceso inflamatorio reactivo benigno que incorpora un grupo heterogéneo de procesos proliferativos linfocíticos de predominio $B$ o $T$ que puede simular clínica y morfológicamente un linfoma cutáneo ${ }^{1}$. Fue reportada por primera vez en 1969 por Caro y Helwig ${ }^{2}$ y también se conoce como pseudolinfoma cutáne $0^{3}$, aunque en la literatura se pueden encontrar otras denominaciones como linfocitoma cutis, pseudolinfoma de Spiegler Fendt y linfoplasia cutánea, que se refieren a un subtipo relativamente común de $\mathrm{HLC}$ caracterizado por un patrón de crecimiento folicular con predominio de células $B^{1,4,5}$.

Su importancia clínica radica en el diagnóstico diferencial con el linfoma cutáneo, que en numerosos casos puede resultar imposible 0 al menos dificultoso ${ }^{1,5}$.

\section{Etiopatogenia}

En la mayoría de los casos se desconoce la etiología, sin embargo, se han descrito numerosas asociaciones que incluyen picaduras de insecto, fármacos, vacunas, tatuajes e infecciones por herpes zóster, Borrelia burgdorferi y Leishmania donovani ${ }^{5,6}$. Los fármacos más frecuentemente asociados son los anticonvulsivantes, aunque también se han reportado casos secundarios a bloqueadores de los canales de calcio, losartán, metotrexato, tamoxifeno y ciclosporina ${ }^{7-9}$.

Se ha planteado la posibilidad de que la HLC realmente corresponda a una etapa temprana de linfoma cutáneo ${ }^{10}$, lo que se sustenta principalmente en el reporte de casos clínicos utilizando como modelo la asociación de $\mathrm{H}$. pylorii con progresión a linfoma de tejido linfoide asociado a mucosa gástrica (MALT) por estimulación antigénica sostenida ${ }^{1,11}$. Considerando lo anterior, si bien muchos de estos casos se han re-diagnosticado como linfomas tras estudios posteriores, la recomendación actual tiende a suspender precozmente el estímulo antigénico ${ }^{1,12}$.

\section{Clasificación}

A diferencia del linfoma cutáneo, no existen en la actualidad criterios unificados para la clasificación de la HLC1 y se clasifica histopatológicamente de manera amplia en relación a su población linfoide ${ }^{13}$, el patrón de infiltración (nodular 0 difuso) y los hallazgos clínicos asociados ${ }^{14}$. Mientras los pseudolinfomas $T$ son un grupo heterogéneo de entidades clínico-patológicas que incluyen la dermatitis linfomatoide de contacto, infiltración linfocítica secundaria a fármacos y el reticuloide actínico, entre otras, caracterizadas por un infiltrado linfocitario denso, en banda en la dermis superior ${ }^{1,14 ;}$; el pseudolinfoma de células $B$ representa una variedad diagnóstica distinta, utilizándose diversos términos para referirse a ella: linfocitoma cutis, pseudolinfoma de Spiegler-Fendt, linfadenosis benigna cutis y linfoplasia cutánea ${ }^{1}$ y se pueden reconocer algunos subtipos clínicos como el nodular secundario a infestación persistente por artrópodos o el inducido por vacunas ${ }^{1,4}$. Debido al advenimiento de los estudios inmunohistoquímicos se ha podido determinar que algunos infiltrados de células B considerados pseudolinfomas B son en realidad linfomas cutáneos de bajo grado, conocidos como linfomas de zona marginal $l^{1,15}$.

\section{Clínica}

La HLC se ha visto tanto en adultos como niños y al parecer es 2 a 3 veces más frecuente en mujeres que en hombres y más frecuente en menores de 40 años ${ }^{5,16}$. Clínicamente se presenta como agrupaciones de pápulas firmes, que pueden agruparse y formar placas violáceas o rosadas, nódulos o tumores. Se ubican preferentemente en la cara, aunque pueden aparecer en cualquier otra región. Si bien la mayoría de las veces son asintomáticas, los pacientes buscan tratamiento principalmente por razones cosméticas. La HLC puede estar asociada con adenopatías regionales, pero lo más frecuente es que no se asocien a otros hallazgos ${ }^{16}$. El curso de esta patología es benigno, desapareciendo las lesiones de manera espontánea en el rango de semanas a meses, con posibilidades de recurrencia e incluso progresión a linfoma, como ya se decribió previamente.

\section{Histopatología}

El diagnóstico se realiza mediante el estudio histopatológico e inmunohistoquímico. En el examen histológico, lo habitual es la presencia de folículos linfoides múltiples con infiltración densa de linfocitos maduros en dermis superficial y media. La zona interfolicular presenta una mezcla de linfocitos con histiocitos, y algunos eosinófilos y células gigantes multinucleadas. Los centros germinales con macrófagos de cuerpo teñible son frecuentes ${ }^{16}$. La disposición en $V$ del infiltrado linfoide junto con la presencia de una Zona de Grenz (banda de colágeno que separa epidemis basal de elemento anormal dérmico) entre el infiltrado linfoide y la epidermis y anexos cutáneos constituye un elemento diagnóstico útil para dife- 
renciarlo de proliferaciones linfoides neoplásicas ${ }^{14}$. Dado que muchas de estas características también se presentan en los linfomas, se propone la diferenciación entre HLC y linfomas a raíz de la presentación con linfocitos policlonales reactivos en la primera, que generalmente consiste en un infiltrado compuesto por CD8 y CD4 en la periferia y LB en los centros germinales y monoclonales en los segundos. El estudio inmunohistoquímico con determinación de CD3, CD10, CD20 y LCA, marcadores de linfoma, son útiles para el diagnóstico diferencial ${ }^{14}$. La determinación de BCL-2, un modulador de la apoptosis, apoya el diagnóstico de linfoma cuando se encuentra sobre expresado, mientras que la sobre expresión de BCLI6 orienta a benignidad ${ }^{17}$. También se plantea como marcador la presencia de restricción de cadenas livianas kappa/lambda, presente en linfomas y no en $\mathrm{HLC}^{18}$.

\section{Diagnóstico diferencial}

El diagnóstico diferencial de esta patología incluye cuadros inflamatorios, tumorales benignos y malignos. Dada su diversa asociación con cuadros como picaduras de insecto, reacciones locales a vacunas y otros descritos previamente, la sospecha clínica inicial se basa en el descarte de éstos. Dado su pronóstico, implicancias terapéuticas y la apariencia anátomopatológica muchas veces indistinguible con el pseudolinfoma, el principal diagnóstico diferencial es el linfoma cutáneo, por lo que deben incluirse técnicas de inmunohistoquímica y métodos biomoleculares durante el estudio diagnóstico ${ }^{1,5,19}$.

Desde el punto de vista clínico debemos tener en cuenta que las lesiones producidas por la HLC son sumamente variables, por lo que vamos a plantear nuestros posibles diagnósticos diferenciales caso a caso. Tomando como punto de partida la lesión elemental (pápulas eritematosas asintomáticas) y los antecedentes personales de nuestra paciente, vamos a proponer los siguientes diagnósticos diferenciales:

- Liquen estriado: Dermatosis inflamatoria autoinvolutiva de etiología desconocida. Se caracteriza por pápulas eritematosas, de superficie plana y brillante que siguen una distribución lineal (líneas de Blaschko). Suelen ubicarse en brazos, piernas y cuello, sin embargo pueden comprometer el tronco en algunos casos. Generalmente afecta personas entre los 5 y 15 años, y es más frecuente en mujeres. A pesar que las lesiones pueden llegar a ser similares a la HLC, las lesiones del liquen estriado tienden a la regresión espontánea alrededor del año de evolución, lo que se aleja del cuadro de nuestra paciente.

- Liquen amilode: Es un tipo de amiloidosis cutánea localizada, derivada del depósito de material proteico amiloide en la matriz extracelular, suele afectar personas entre la $4^{\text {ta }} y$ la $5^{\text {ta }}$ década de la vida, y se caracteriza por el desarrollo de placas formadas por pápulas de 1-3 mm de color café-amarillentas o grisáceas, generalmente poligonales, firmes e indoloras, secundarias al rascado crónico. La ausencia de prurito en el caso de nuestra paciente nos aleja de esta entidad, ya que es el principal factor gatillante para la aparición de las lesiones cutáneas.

- Papulosis linfomatoide cutánea: Es una variedad de linfoma T cutáneo, de bajo grado de malignidad que se presenta habitualmente en adultos jóvenes, pero que también puede llegar a afectar a niños. Se caracteriza por una erupción generalizada de pápulas o pequeños nódulos eritematosos que afectan al tronco y a las áreas proximales de las extremidades. Suele tener una regresión espontánea en meses o años, pero también puede progresar a linfoma maligno. La característica principal de esta entidad es la coincidencia de lesiones en distintos estadios evolutivos en un mismo paciente (nódulos, úlceras, costras), por lo que contrastándolo con las lesiones de nuestra paciente, se vuelve un diagnóstico improbable.

- Liquen plano: Dermatosis inflamatoria mucocutánea de etiología poco clara. Se caracteriza por la aparición de pápulas, planas, purpúricas, poligonales, muy pruriginosas, que se distribuyen generalmente en superficies flexoras de muñecas y antebrazos, región lumbar y piernas. Puede también comprometer la mucosa oral y la superficie ungueal. Suelen tener una evolución autolimitada, persistiendo alrededor de un año sin tratamiento, y con alta tasa de recurrencia. Aunque las lesiones pudieran llegar a parecerse a las de la HLC, la clínica (prurito intenso), y un adecuado examen dermatológico debieran ser suficientes para descartar este cuadro.

\section{Tratamientos}

Considerando el curso benigno de esta enfermedad, el tratamiento muchas veces será la observación y si se conoce, la suspensión del estímulo sostenido ${ }^{1,16}$. Frente a lesiones persistentes o recurrentes y tras la realización de los estudios correspondientes, se ha considerado la utilidad de diversas terapias; entre las que se encuentran la escisión quirúrgica, criocirugía, radiación local, corticoides intralesionales, antibióticos, antimaláricos, interferones, citotóxicos, inmunosupresores y fototerapia PUVA ${ }^{4,14,20-25}$. En estudios de casos se ha demostrado la utilidad de Talidomida ${ }^{21}$, Tacrolimus ${ }^{4}$, terapia fotodinámica ${ }^{25}$ y más recientemente Imiquimod $5 \%$ (5 aplicaciones/semana por 6 semanas) con buena respuesta en un caso recalcitrante de pseudolinfoma ${ }^{22}$. 


\section{Comentario final}

Lo interesante de este caso es el antecedente le una lesión de aparición muy precoz (8 años de edad) con un patrón de distribución zosteriforme siguiendo las lineas de Blaschko no descrito previamente en la literatura. Llama la atención el resultado histopatológico de la primera biopsia (2003) compatible con una reacción tardía a picadura de insecto como posible agente gatillante. Tuvimos la suerte de poder controlar a la paciente después de 12 años y observar la evolución progresiva de las lesiones, actualmente con un patrón de distribución más evidente. Considerando que la HLC representa una reacción inmune local benigna, la aparición precoz y evolución progresiva crónico-recurrente de nuestra paciente, nos obliga a un seguimiento y control periódico clínico e histopatológico con nuevas biopsias para detectar precózmente una posible transformación maligna.

\section{Referencias bibliográficas}

1. Bergman R. Pseudolymphoma and cutaneous lymphoma: facts and controversies. Clinics in dermatology. 2010;28(5):568-74. Epub 2010/08/28.

2. Caro WA, Helwig HB. Cutaneous lymphoid hyperplasia. Cancer. 1969;24(3):487-502. Epub 1969/09/01.

3. Dragonetti E, Cianchini G, Mastrangelo L, Mellone P, Baldi A. Cutaneus pseudolymphoma: a case report. In Vivo. 2004;18(5):549-51. Epub 2004/11/05.

4. El-Dars LD, Statham BN, Blackford S, Williams N. Lymphocytoma cutis treated with topical tacrolimus. Clinical and experimental dermatology. 2005;30(3):305-7. Epub 2005/04/06.

5. Hasan M, Shahid M, Varshney M, Mubeen A, Gaur K. Idiopathic lymphocytoma cutis: a diagnostic dilemma. BMJ case reports. 2011;2011. Epub 2011/01/01.

6. Moulonguet I, Garcon N, Rivet J, Cavelier-Balloy B. Nodule developing over a tattoo: challenge. Cutaneous lymphoid hyperplasia (pseudolymphoma). The American Journal of dermatopathology. 2014;36(1):88-9, 101-2. Epub 2014/01/24.

7. Foley C, Leonard N, Wynne B. Cutaneous pseudolymphoma: a rare side effect of cyclosporine. Journal of the American Academy of Dermatology. 2015;72(3):e85-6. Epub 2015/02/18.

8. Viraben R, Lamant L, Brousset P. Losartan-associated atypical cutaneous lymphoid hyperplasia. Lancet. 1997;350(9088):1366. Epub 1997/11/20.

9. Magro CM, Crowson AN. Drugs with antihistaminic properties as a cause of atypical cutaneous lymphoid hyperplasia. Journal of the American Academy of Dermatology. 1995;32(3):419-28. Epub 1995/03/01.
10. Nihal M, Mikkola D, Horvath N, Gilliam AC, Stevens SR, Spiro TP, et al. Cutaneous lymphoid hyperplasia: a lymphoproliferative continuum with lymphomatous potential. Human pathology. 2003;34(6):617-22. Epub 2003/06/27.

11. Sangueza OP, Yadav S, White CR, Jr., Braziel RM. Evolution of B-cell lymphoma from pseudolymphoma. A multidisciplinary approach using histology, immunohistochemistry, and Southern blot analysis. The American Journal of dermatopathology. 1992;14(5):408-13. Epub 1992/10/11.

12. Goodlad JR, Davidson MM, Hollowood K, Batstone P, Ho-Yen DO. Borrelia burgdorferi-associated cutaneous marginal zone lymphoma: a clinicopathological study of two cases illustrating the temporal progression of B. burgdorferi-associated B-cell proliferation in the skin. Histopathology. 2000;37(6):501-8. Epub 2000/12/21.

13. Prabhu V, Shivani A, Pawar VR. Idiopathic cutaneous pseudolymphoma: An enigma. Indian dermatology online journal. 2014;5(2):224-6. Epub 2014/05/27.

14. Tapia O KS, Valenzuela Y. Hiperplasia cutánea linfoide (linfocitoma cutis) secundaria a fármacos: hallazgos

15. clinicos y morfológicos. Int J Morphol. 2012;30(1):5. Epub 03/2012.

16. LeBoit PE. Cutaneous lymphocytic infiltrates: let's get real. The American Journal of dermatopathology. 2005;27(2):182-4. Epub 2005/03/31.

17. Lackey JN, Xia Y, Cho S, Sperling LC. Cutaneous lymphoid hyperplasia: a case report and brief review of the literature. Cutis. 2007;79(6):445-8. Epub 2007/08/24.

18. Terada T. Cutaneous pseudolymphoma: a case report with an immunohistochemical study. International journal of clinical and experimental pathology. 2013;6(5):966-72. Epub 2013/05/03.

19. Boudova L, Kazakov DV, Sima R, Vanecek T, Torlakovic E, Lamovec J, et al. Cutaneous lymphoid hyperplasia and other lymphoid infiltrates of the breast nipple: a retrospective clinicopathologic study of fifty-six patients. The American Journal of dermatopathology. 2005;27(5):375-86. Epub 2005/09/09.

20. Cerroni LG, K. Kerl, H. An illustrated guide to skin lymphomas (2nd ed.)2004. $21 \mathrm{p}$.

21. Olson LE, Wilson JF, Cox JD. Cutaneous lymphoid hyperplasia: results of radiation therapy. Radiology. 1985;155(2):507-9. Epub 1985/05/01.

22. Benchikhi H, Bodemer C, Fraitag S, Wechsler J, Delfau-Larue MH, Gounod $\mathrm{N}$, et al. Treatment of cutaneous lymphoid hyperplasia with thalidomide: report of two cases. Journal of the American Academy of Dermatology. 1999;40(6 Pt 1):1005-7. Epub 1999/06/12.

23. Baumgartner-Nielsen J, Lorentzen $\mathrm{H}$. Imiquimod 5\%: a successful treatment for pseudolymphoma. Acta dermato-venereologica. 2014;94(4):469. Epub 2013/11/12.

24. Singletary HL, Selim MA, Olsen E. Subcutaneous interferon alfa for the treatment of cutaneous pseudolymphoma. Archives of dermatology. 2012;148(5):572-4. Epub 2012/07/12.

25. Stoll DM. Treatment of cutaneous pseudolymphoma with hydroxychloroquine. Journal of the American Academy of Dermatology. 1983:8(5):696-9. Epub 1983/05/01.

26. O'Neill J, Fien S, Zeitouni NC. ALA-PDT for the treatment of cutaneous pseudolymphoma: a case report. Journal of drugs in dermatology : JDD. 2010;9(6):688-9. Epub 2010/07/22. 